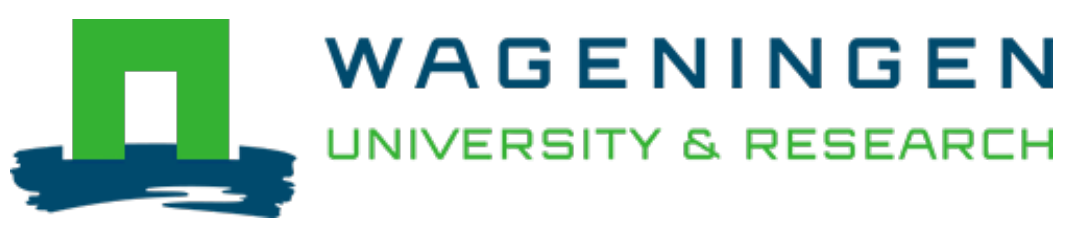

\title{
Concurrent design of product and supply chain architectures for modularity and flexibility : process, methods, and application
}

International Journal of Production Research

Gan, Thiam Soon; Steffan, Moritz; Grunow, Martin; Akkerman, Renzo

https://doi.org/10.1080/00207543.2021.1886370

This article is made publicly available in the institutional repository of Wageningen University and Research, under the terms of article $25 \mathrm{fa}$ of the Dutch Copyright Act, also known as the Amendment Taverne. This has been done with explicit consent by the author.

Article 25 fa states that the author of a short scientific work funded either wholly or partially by Dutch public funds is entitled to make that work publicly available for no consideration following a reasonable period of time after the work was first published, provided that clear reference is made to the source of the first publication of the work.

This publication is distributed under The Association of Universities in the Netherlands (VSNU) 'Article $25 \mathrm{fa}$ implementation' project. In this project research outputs of researchers employed by Dutch Universities that comply with the legal requirements of Article $25 \mathrm{fa}$ of the Dutch Copyright Act are distributed online and free of cost or other barriers in institutional repositories. Research outputs are distributed six months after their first online publication in the original published version and with proper attribution to the source of the original publication.

You are permitted to download and use the publication for personal purposes. All rights remain with the author(s) and / or copyright owner(s) of this work. Any use of the publication or parts of it other than authorised under article $25 \mathrm{fa}$ of the Dutch Copyright act is prohibited. Wageningen University \& Research and the author(s) of this publication shall not be held responsible or liable for any damages resulting from your (re)use of this publication.

For questions regarding the public availability of this article please contact openscience.library@,wur.nl 


\section{Concurrent design of product and supply chain architectures for modularity and flexibility: process, methods, and application}

Thiam-Soon Gan, Moritz Steffan, Martin Grunow \& Renzo Akkerman

To cite this article: Thiam-Soon Gan, Moritz Steffan, Martin Grunow \& Renzo Akkerman (2021): Concurrent design of product and supply chain architectures for modularity and flexibility: process, methods, and application, International Journal of Production Research, DOI: 10.1080/00207543.2021.1886370

To link to this article: https://doi.org/10.1080/00207543.2021.1886370

View supplementary material $\llbracket$

曲 Published online: 22 Mar 2021.

Submit your article to this journal $\pi$

Џ Article views: 94

View related articles $₫$

View Crossmark data $\nearrow$ 


\title{
Concurrent design of product and supply chain architectures for modularity and flexibility: process, methods, and application
}

\author{
Thiam-Soon Gan ${ }^{\mathrm{a}}$, Moritz Steffan ${ }^{\mathrm{b}}$, Martin Grunow (1) a and Renzo Akkerman (1) ${ }^{\mathrm{c}}$ \\ aProduction and Supply Chain Management, TUM School of Management, Technical University of Munich, Munich, Germany; ${ }^{\mathrm{b}}$ Invenox GmbH, \\ Garching, Germany; ' Operations Research and Logistics group, Wageningen University, Wageningen, The Netherlands
}

\begin{abstract}
Product design and supply chain design are two key determinants of company competitiveness. However, they follow different design objectives and thus require a systematic trade-off. Although methodologies for product design and supply chain design are well established within each domain in research and industry, an integrated methodology that bridges both design domains is still lacking. Based on a recently introduced concurrent product and supply chain design process, we contribute to this underdeveloped research area with a generic approach towards exploring design tradespace. We introduce a detailed operational process for the concurrent design of product and supply chain architectures. To apply this generic process to the specific trade-off between the product-related objective of modularity and the supply-chain-related objective of sourcing flexibility, we also develop new methods for key steps of the process. We demonstrate the application of the process and the developed methods using an industrial case study of a new product (electricvehicle battery module). The case shows that our methodology was able to structure the concurrent design process. It hereby ensured an efficient trade-off and led to high-quality designs.
\end{abstract}

ARTICLE HISTORY

Received 18 August 2020

Accepted 24 January 2021

\section{KEYWORDS}

Concurrent design; product

modularity; sourcing

flexibility; DSM;

electric-vehicle battery

\section{Introduction}

Manufacturing companies can be classified by product type: niche products, differentiated products, and commodities. Niche product manufacturers' competitiveness depends mainly on product design (PD) attributes such as aesthetics, functional performance, and customisability. In contrast, commodity manufacturers' competitiveness depends mainly on supply chain design (SCD) attributes such as cost, flexibility, and lead time. As most companies produce differentiated products, they have to consider both types of design attributes to ensure overall competitiveness. This reflects the key ideas in the seminal work by Fisher (1997), highlighting the importance of matching the right supply chain (SC) to the product. While this is relatively easy for niche and commodity products, it is less so for differentiated products due to the lack of trade-off methodologies between PD and SCD (Gan and Grunow 2016).

PD and SCD are strategic decisions in two central but different functions in manufacturing companies. Traditionally, the product is designed first, and the appropriate SC structure is then chosen with the typical aim of finding the best possible trade-off between efficiency and responsiveness (Fisher 1997). However, a sequential approach unduly limits the design space for SCD. For example, the overwhelming focus on a highly customisable PD in the development of the NH90 European military helicopter led to an inferior SCD, consisting of a myriad of small, inefficient, and redundant suppliers. This SCD contributed to more than ten years of delivery delays and cost overruns (Uiterwijk, Soeters, and van Fenema 2013). More recently, CNBC (2018) reported that the decision of electric car manufacturer Tesla to use type 2170 battery cells (optimised for high energy density and supplied by a single supplier) instead of the commonly available but less efficient type 18650 battery cells resulted in severe SC delays due to its single-sourcing strategy. In both examples, the emphasis on PD undermined the efficiency and the responsiveness of the SCD.

\subsection{Concurrent product and supply chain design}

Concurrent product and supply chain design (CPSCD), in contrast, explores the two design spaces

\footnotetext{
CONTACT Thiam-Soon Gan thiam_soon.gan@tum.de TUM School of Management, Technical University of Munich, Arcisstrasse 21, 80333 Munich, Germany

(-) Supplemental data for this article can be accessed here. https://doi.org/10.1080/00207543.2021.1886370
} 
simultaneously. Using this method, SC efficiency and SC responsiveness can be considered earlier in the design process, potentially at the expense of the $\mathrm{PD}$. The understanding of such trade-offs between PD and SCD is so far underdeveloped (Pashaei and Olhager 2015). Increasingly complex products and SCs do however require systematic concurrent approaches to characterise the tradespace between optimal PD and optimal SCD. Here, the design tradespace is defined as the set of combinations of product and SC designs optimised with varying relative importance of PD and SCD objectives. Research on such methodologies is unfortunately lacking (Ellram and Stanley 2008; Khan and Creazza 2009; Gokhan, Needy, and Norman 2010; Baud-Lavigne, Agard, and Penz 2012; Gan and Grunow 2016; Yao and Askin 2019).

In the development of CP-SCD approaches, different design levels could be addressed. PD can be done at architectural, detailed, or dynamic level while SCD can be done at strategic, tactical, or operational level. Any CP-SCD method must address attributes from similar hierarchy levels (Gan and Grunow 2016) and must be able to allow design attributes of both domains to be commensurable, aggregable, and compensable (Colson and de Bruyn 1989; Guitouni and Martel 1998). The higher the hierarchy level, the higher the impact on the design of the product and its SC. We therefore focus on the architectural-strategic level. Amongst the design attributes on this level, modularity and flexibility are widely considered to be the most important for both domains (Fine 1998; Simchi-Levi 2013).

\subsection{Product and supply chain modularity}

In $\mathrm{PD}$, modularity is widely considered to be the most important architectural attribute because of the distinct advantages that it provides (e.g. configurability) and enables (e.g. flexibility, replaceability) (Ulrich 1995; Mikkola and Gassmann 2003). Modularity enables flexibility for product reconfiguration as well as replaceability for repairs and upgrades, both of which affect product lifecycle performance (Ross, Rhodes, and Hastings 2008). Product modularity is well-documented in e.g. Mikkola and Gassmann (2003), Gershenson, Prasad, and Zhang (2004), Hölttä, Suh, and de Weck (2005), and Jung and Simpson (2017).

The concept of modularity can also be applied to SCD (Pashaei and Olhager 2015). SCD modularity is also considered one of the most important strategic design attributes (Duclos, Vokurka, and Lummus 2003; Zhang, Huang, and Rungtusanatham 2008; Khan and Creazza 2009; Simchi-Levi 2013; Jayaram and Vickery 2018). It is important for lowering transaction costs by simplifying
SC structures and reducing associated complexity. Furthermore, it increases efficiency by enabling standardisation of production, shorter lead times, and reduced inventories (Feng and Zhang 2014).

SC modularity leads to sourcing flexibility, which is defined as the ability to reconfigure the SC according to supply and demand changes (Duclos, Vokurka, and Lummus 2003). This is vital for the mitigation of risks associated with demand fluctuations, SC disruptions, and supplier quality problems (Tang and Tomlin 2008; Saleh, Mark, and Jordan 2009; Simchi-Levi 2013). Moreover, sourcing flexibility has far-reaching implications for manufacturing companies: it enables multiple sourcing, which in turn ensures sourcing price stability and the sustainability of a competitive, non-monopolistic industrial ecosystem. For example, automotive companies commonly use different suppliers for the same components in different final products. This enables these companies to mitigate disruption risks. Also, it improves the bargaining power over suppliers (Henkel and Hoffmann 2018).

\subsection{Sourcing flexibility}

The role of sourcing flexibility in supplier selection and its dependency on the buyer-supplier relationship has received much attention in past research. De Boer, Labro, and Morlacchi (2001) reviewed methods for supplier selection and sourcing decisions, highlighting the complexity of the procurement process before supplier selection and sourcing decisions are made. They also argue that the purchasing situation is an important factor for selecting the right decisional method for sourcing strategy. Gosling, Purvis, and Naim (2010) highlight the lack of consideration of SC flexibility in supplier selection research. They suggest that SC flexibilities are key criteria for supplier selection and supplier relationship development. While the literature stresses the impact of many criteria on sourcing decisions, they consider product architectures fixed, hereby overlooking the impact of product architectures on the sourcing decisions.

Decisions on product modularity and SC modularity are strongly interconnected and have an impact on sourcing flexibility. For instance, in PD, it might be beneficial to combine some components in a module, but it might not be possible to source the resulting module from a sufficient number of suppliers, thus reducing sourcing flexibility. Similarly, in SCD, simple SC structures with low transaction costs are preferred, but they may lead to fewer and larger modules, which in turn may lead to combinations of components that are impossible to integrate in a product design. Hence, CP-SCD approaches should facilitate discussions between product designers and SC designers early in the design process. 


\subsection{Contributions}

In this paper, we pursue four aims. Firstly, we aim at building an understanding of the interaction between PD and SCD by showing how the tradespace between the optimal PD and the optimal SCD can be generically characterised at the architectural-strategic level. Secondly, we aim to develop a detailed CP-SCD process for the generation of the design tradespace. Thirdly, we aim to introduce new methods to generate PD and SCD architectures. For the PD architecture a clustering method is used in the creation of product modules. The SCD method focusses on supplier selection and sourcing flexibility, which need to be considered early in the CP-SCD process. These PD and SCD methods specifically focus on modularity and flexibility but could be adapted to consider other attributes such as various cost factors, responsiveness, or environmental footprints. Finally, we aim to demonstrate the application of the CP-SCD process using an industrial case study (of a new electric-vehicle battery) that characterises a typical CP-SCD situation. With this, we also aim to show how the systematic exploration of the design space can lead to a range of solutions that provide a better understanding of the trade-off between PD and SCD objectives.

\subsection{Paper organisation}

This paper is structured as follows. In Section 2, we provide a literature review on methodologies for CP-SCD, architectural PD, and strategic SCD. In Section 3, we introduce the case study (electric vehicle battery system). In Section 4, we use introduce detailed procedures for architectural-strategic design in the CP-SCD process and specific clustering methods using data from the case study. Moreover, we explain the key concepts for the characterisation of design tradespace for CP-SCD, a cornerstone of this paper. Finally, in Section 5, we discuss our contributions, limitations, and the potential improvements to our methodology for future research.

\section{Literature review}

\subsection{Architectural design of products and supply chains}

Methods for architectural PD are well established in research and industry. Some examples are Axiomatic Design Method, Object Process Methodology, and Design Structure Matrix (DSM) (Suh 1998; Dori 2002; Fixson 2005; Tilstra, Seepersad, and Wood 2012; Jung and Simpson 2017). DSM is more commonly used in industry because of its flexibility in modelling different $\mathrm{PD}$ and non-PD attributes and visualising the clustering results (e.g. Behncke, Walter, and Lindemann 2014). DSM can either use binary or numerical values in a matrix to map functional or relational interactions between parts. In industry, the functionalities of DSM have been further developed and used in specialised software tools (e.g. METUS) that provide advanced user interfaces as well as connections to data analytics tools.

Several methods of strategic SCD exist. Examples of qualitative methods are frameworks for core-competence analysis, make-or-buy analysis, and supplier integration to support decision making in strategic SCD (Fine and Whitney 1996; De Boer, Labro, and Morlacchi 2001; Novak and Eppinger 2001; Noori and Georgescu 2008; Gosling, Purvis, and Naim 2010). Quantitative methods of strategic SCD are typically model-based methods that mathematically link interactions between SCD attributes for optimisation (Beamon 1998; Meixell and Gargeya 2005; Melo, Nickel, and Saldanha-da-Gama 2009).

Both PD and SCD are fields with a large body of literature that has introduced various methods dedicated to the architectural-strategic design level of the individual field. In the following, we discuss research on concurrent approaches.

\subsection{Concurrent product and supply chain design}

CP-SCD is recognised by many as an important research area and a vital industrial capability (Blackhurst, Wu, and O'Grady 2005; Zhang, Huang, and Rungtusanatham 2008; ElMaraghy and Mahmoudi 2009; Gokhan, Needy, and Norman 2010; Chiu and Okudan 2014). Despite this recognition, it is still an emerging research field, which is also clear from three recent review papers that highlight the state-of-the-art research on CP-SCD (Pashaei and Olhager 2015; Gan and Grunow 2016; Yao and Askin 2019). Published around the same time, Pashaei and Olhager (2015) and Gan and Grunow (2016) find that there is limited understanding of how design decisions for PD and SCD interact with each other. While the impact of PD on SCD is well studied, the impact of SCD on PD is unexplored, and little work is done on the development of methodologies for CP-SCD. Pashaei and Olhager (2015) further note that there is a lack of case studies in CP-SCD. Past research focusses on extreme PD cases (either modular or integral) and the design tradespace between these extremes is underexplored. More recently, Yao and Askin (2019) review and identify different representation schemes (e.g. BOM, DSM) used for CP-SCD and note a lack of studies on matrix representations (e.g. DSM) and clustering efficiency. Gan and Grunow (2016) additionally summarise their findings in a conceptual framework, synthesising existing design hierarchical structures from both domains, classifying 
the different types of design attributes, and laying the foundation for fundamental propositions on the quality of CP-SCD methodologies. Using these propositions, they further propose a conceptual CP-SCD process at architectural-strategic level. However, this conceptual process remains to be operationalised and applied to industrial cases, which are the aims of our current paper.

CP-SCD-related frameworks that analyse the relationship between PD and SCD attributes exist. Fine and Whitney (1996) include relationships between PD attributes (e.g. product architecture, modularity) and SCD attributes (e.g. make-or-buy decision). Fine (1998) extends this by linking product, process, and SC designs. Appelqvist, Lehtonen, and Kokkonen (2004) map data exchange processes between the PD and SCD activities of an aerospace company. Pero et al. (2010) analyse the relationships between PD attributes (modularity, variety, innovativeness) and SCD attributes (structure, configuration) in several case studies. Gokhan, Needy, and Norman (2010) introduce the process of Design for Supply Chain as an improvement to sequential design processes. This contributes to methodological CP-SCD research by analysing the procedural aspect of CP-SCD. Although all these frameworks provide multifaceted perspectives on CP-SCD methodology, they do not specify approaches for the exploration of design tradespace and for design trade-off, which are imperative for ensuring the quality of design decision.

While previous literature does not provide CP-SCD methodologies that explore the interaction between $\mathrm{PD}$ and SCD, literature on Multi-Criteria Decision Analysis (MCDA) does provide a basis for the development of such methodologies. CP-SCD methodology needs to identify the interdependency between PD and SCD attributes and provide an approach to balance between conflicting design objectives of PD and SCD. For the purpose of linking these design domains and their attributes, the principles of compensability, commensurability, and aggregation between PD and SCD attributes need to be ensured (Colson and de Bruyn 1989; Guitouni and Martel 1998). Adherence to these principles allows the use of MCDA methods (Ehrgott, Figuira, and Greco 2010) to model and search for optimal solutions in the tradespace.

\subsection{Key findings of the literature review}

In summary, we identify the following literature gaps:

- Past research focussed only on the impact of PD on SCD. There is a lack of understanding of the impact of SCD on PD. It lacks the more comprehensive exploration of the design tradespace for different module configurations formed under product and supply chain objectives.

- Past frameworks for CP-SCD are of a conceptual nature and are not sufficiently specific to be applied in practice. Methodologies that systematically and hierarchically integrate PD and SCD methods are lacking. Particularly at the architectural-strategic level, methodologies supporting a systematic assessment of the design tradespace are missing.

- There is an abundance of qualitative and quantitative methods for product architectural and strategic SCD. However, they are dedicated to the specific domain. Matrix representations, widely used in $\mathrm{PD}$, have not been developed sufficiently for use in CP-SCD. Also, even though MCDA methods are well established, they have not been used to bridge the domains.

- Finally, there is a lack of case studies in CP-SCD that explore the design tradespace between product supply chain (P-SC) system architectures.

Our review thus highlights the immature status of literature on CP-SCD. These findings motivate us to develop a detailed CP-SCD process and methods that together address the aforementioned issues and translate conceptual frameworks into an operational methodology for the architectural-strategic design level. We also introduce a case study to demonstrate the application of the CP-SCD process.

\section{Introduction to the case study}

In this section, we introduce the case study that is used to explain the procedure and methods of the operationalised CP-SCD process. The case study focusses on a battery system named Conchifera, which is being developed by a Munich-based company (Invenox $\mathrm{GmbH}$ ). Conchifera has been selected as it is a differentiated product that is still under development. The company has a green-field SC situation that suits the application of the CP-SCD process. The objective of this case study is to find the best design trade-off between the product design architecture (PDA) and the supply chain architecture (SCA) of Conchifera using modularity as the common design attribute.

In general, case study research is useful for research stage exploration, theory building, theory testing, as well as theory refinement (Voss, Tsikriktsis, and Frohlich 2002). Moreover, case study research is useful when it is relevant to the understanding of the interactions between organisations and methodologies (e.g. Eisenhardt and Graebner 2007; Ketokivi and Choi 2014). Our work is in the early research stages, in which a single case study approach is often chosen since this allows for an 
in-depth analysis (Voss, Tsikriktsis, and Frohlich 2002). Our methodology is applied in its natural setting and its effectivity observed in practice, which is important for evaluating the complex interactions between the different functional (PD and SCD) teams of Invenox when using our methodology.

In the case study, two of the authors participated in numerous meetings, which involved the product designers and SC managers from the company, to observe their discussions and to gather direct feedbacks. All data and company documentation required for our methodology were collected in these meetings. For example, the DSMs, Part-Supplier Matrices (PSM) and design tradespace were created together with the product designers and SC managers.

Conchifera is being developed in pursuit of higher PD and SCD performance (e.g. energy density and assembly cost, respectively). The main advantages of Conchifera are twofold: the scalability of mass assemblies of battery cells with short lead times, and the configurability using common cylindrical cells (type 18650) for diverse applications. These advantages are made possible using a proprietary contact material that connects battery cells, unlike welded connectors used in other battery systems. This material minimises part quantity, weight, and cost. Moreover, it allows faulty cells to be replaced individually and easily to restore the system lifespan as well as ensuring a high level of safety (Hammer et al. 2014).

Conchifera's main components are shown in Figure 1. There is a total of 14 unique parts in the quadruple variant of Conchifera. These components are defined by the functions derived from the PD requirements and can be sourced from different suppliers who have overlapping supply scope. While simpler components (e.g. plastic housing) can be sourced from several suppliers with similar quality and functional performance, it is more difficult for relatively more complex components (e.g. circuit boards).

Some components can only be sourced from a few specialised suppliers. 14 potential suppliers have been identified based on their capabilities and supply scope (Appendix A3, see supplemental data). The battery cells and contact material components are excluded from our case study due to quality and proprietary reasons. Although battery cells are considered commodities, the quality of battery cells from different suppliers can differ significantly. The contact material is a core proprietary component and is therefore manufactured in-house.

\section{Methodology: the CP-SCD process}

\subsection{Overview of the CP-SCD process for architectural design}

Gan and Grunow (2016) derive a conceptual CP-SCD process for modularity design. This process is derived from four propositions for CP-SCD, which are grounded in an extensive literature review. These propositions can be summarised as follows:

(1) A concurrent design process for PD and SCD can bring greater value than a sequential design process.

(2) A concurrent design process should have a balanced inclusion of design attributes from both domains to ensure higher trade-off quality.

(3) A concurrent design process should pursue design trade-offs at the same hierarchical level (starting from the architectural-strategic level) to allow for more accurate modelling of attribute relationships and a higher trade-off quality.

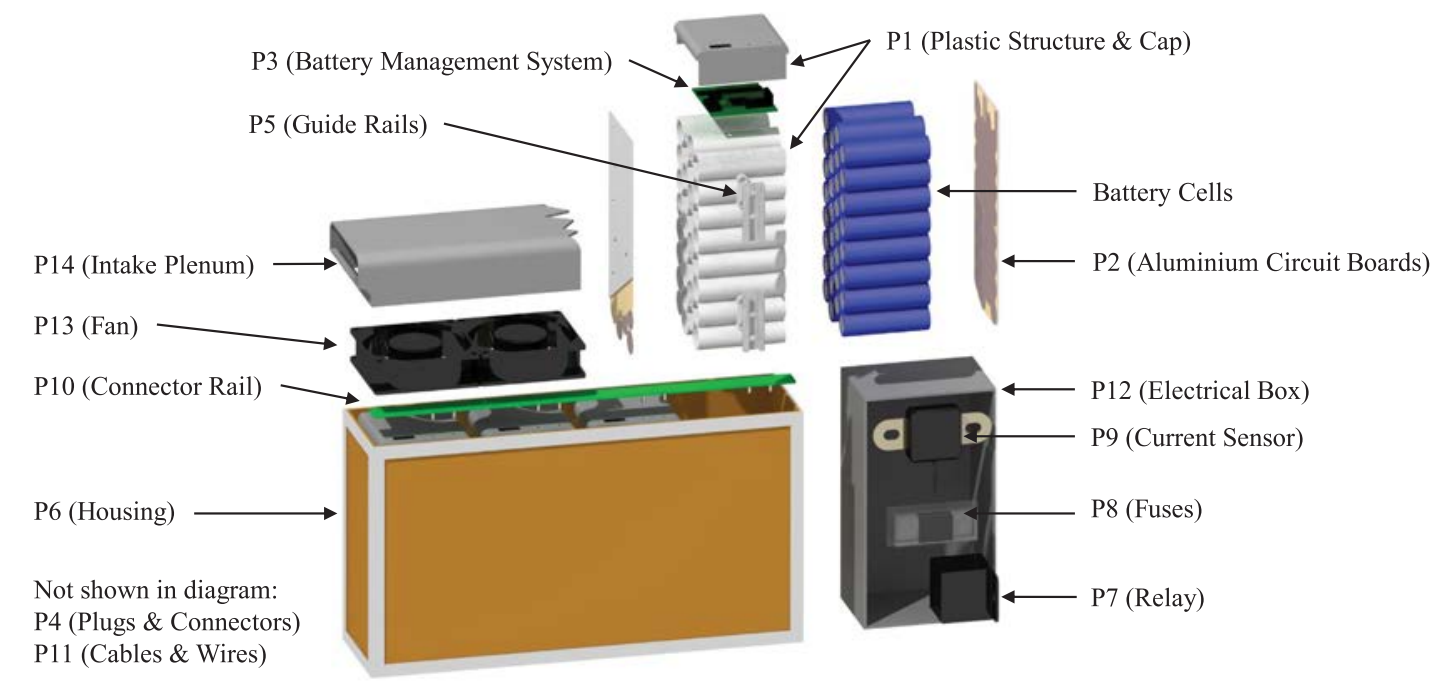

Figure 1. Conchifera electrical vehicle battery system (quadruple variant). 
(4) A concurrent design process should avoid tradeoffs at the lowest hierarchical levels due to the lack of trade-off opportunities between PD and SCD attributes on those levels.

Here, we build on the conceptual model that Gan and Grunow (2016) derived from these propositions and develop a detailed operational process, consisting of three different phases: strategic alignment, concurrent product and SC architectural design, and design trade-off (illustrated in Figure 2 and further described below). The concurrent consideration of PD and SCD in our process results from Proposition 1. The hierarchical, topdown approach of our process, which starts with the architectural-strategic phase before the detailed design phase, follows Proposition 3. The choice of an architectural PD attribute (product modularity) and a strategic SCD attribute (sourcing flexibility) follows Propositions 2 and 4 , as these attributes are at the same, relatively high hierarchical design level, enabling the commensuration, aggregation, and compensation of design attributes. The detailed design level following the three phases of our process is not considered, as trade-off opportunities would be limited on that level and concurrency is not required anymore (following Proposition 4).

Our process is applicable for PD and SCD under different objectives. It has a hierarchical structure with open interfaces to specific methods (the grey boxes in Figure 2). In the remainder of this section, we first discuss the general procedure for each of the three phases. We then develop methods for the specific trade-off between product modularity and SC sourcing flexibility.

In Phase 1, product and SC designers align the product and SC strategies (e.g. target number of modules and required sourcing flexibility) based on the PD and SCD requirements. Examples of product strategy can be found in Ulrich (1995), who focuses on the linkages between product architectures and different PD strategies such as product change, product variety, component standardisation, product performance, and product development management. Examples of SC strategy can be found in Fisher (1997), who focuses on matching the right products to the right SCD. He outlines that functional and innovative products need different types of SCD (i.e. efficient versus responsive). PD and SCD requirements are elicited from the market (e.g. customers, users) and the SC (e.g. suppliers, partners). Next, PD and SC design attributes are defined from the elicited PD and SCD requirements. Established methods for requirement elicitation can be found in Walden et al. (2015).

In Phase 2, the procedures for PD and SCD are described in two concurrent streams. Each stream involves an independent procedure of numerical matrix operations. In contrast to past studies that are mostly sequential (e.g. Nepal, Monplasir, and Famuyiwa 2012; Chiu and Okudan 2014) and that assume the PD to be given (or limited to a very small number of PD options) in the SCD, we explore both PD and SCD concurrently based on the broad requirements resulting from the strategic alignment. Where past studies thus only show the impacts of different PD scenarios on the SCD, we avoid limiting the design trade-offs in this part of the design process. The concurrent design streams in Phase 2 allow designers to build up the product architecture (PDA) and supply chain architecture (SCA) independently and supported their convergence to a commensurable, aggregated, and compensable set of design data structures using matrices (DSM and PSM).

For the product architectural design stream, DSMs are used to map the PD attributes. These DSMs are then weighted based on the prioritisation of the $\mathrm{PD}$ attributes using a suitable MCDA methodology and aggregated to generate the PD-DSM. This stream ends after the generation of the PDA, which represents a clustered PD-DSM (for which a variety of clustering methods may be used).

For the SC architectural design stream, the SCA is first derived. This may be done using a variety of SCD methods and objectives (e.g. costs, speed, or sustainability), possibly resulting in multiple SCAs. However, the SCAs are based on SC attributes other than parts (e.g. supplier, delivery lead times, location, or manufacturing technologies). As SCA representations that allow design trade-offs in relation to the PDA are lacking, our approach introduces a transformation of the SCA into a part-to-part SC-DSM to link the PD and SCD domains. For PD, DSM is an established methodology. For SCD, working with a matrix representation is still uncommon. For this transformation, additional operations using specific methods are required. Note that the main difference between the PD and SCD streams is the procedural sequence. In the product architectural design stream, the architectural design is the last step of the procedure. The DSMs are weighted and aggregated into a PD-DSM and then clustered into a PDA. In the SC architectural design stream, the architectural design is the first step of the procedure. The SCAs are aggregated into an SC-DSM.

Following Propositions 2 and 3, the specific design trade-off we consider in our case (product modularity vs sourcing flexibility) concerns attributes on the same hierarchical level (i.e. the architectural-strategic level). Past studies have often considered design trade-offs between attributes at different hierarchical levels, reducing the quality of the design trade-off in CP-SCD. For example, non-strategic SCD attributes are often traded off against architectural PD attributes (Nepal, Monplasir, and Famuyiwa 2012; Chiu and Okudan 2014). 


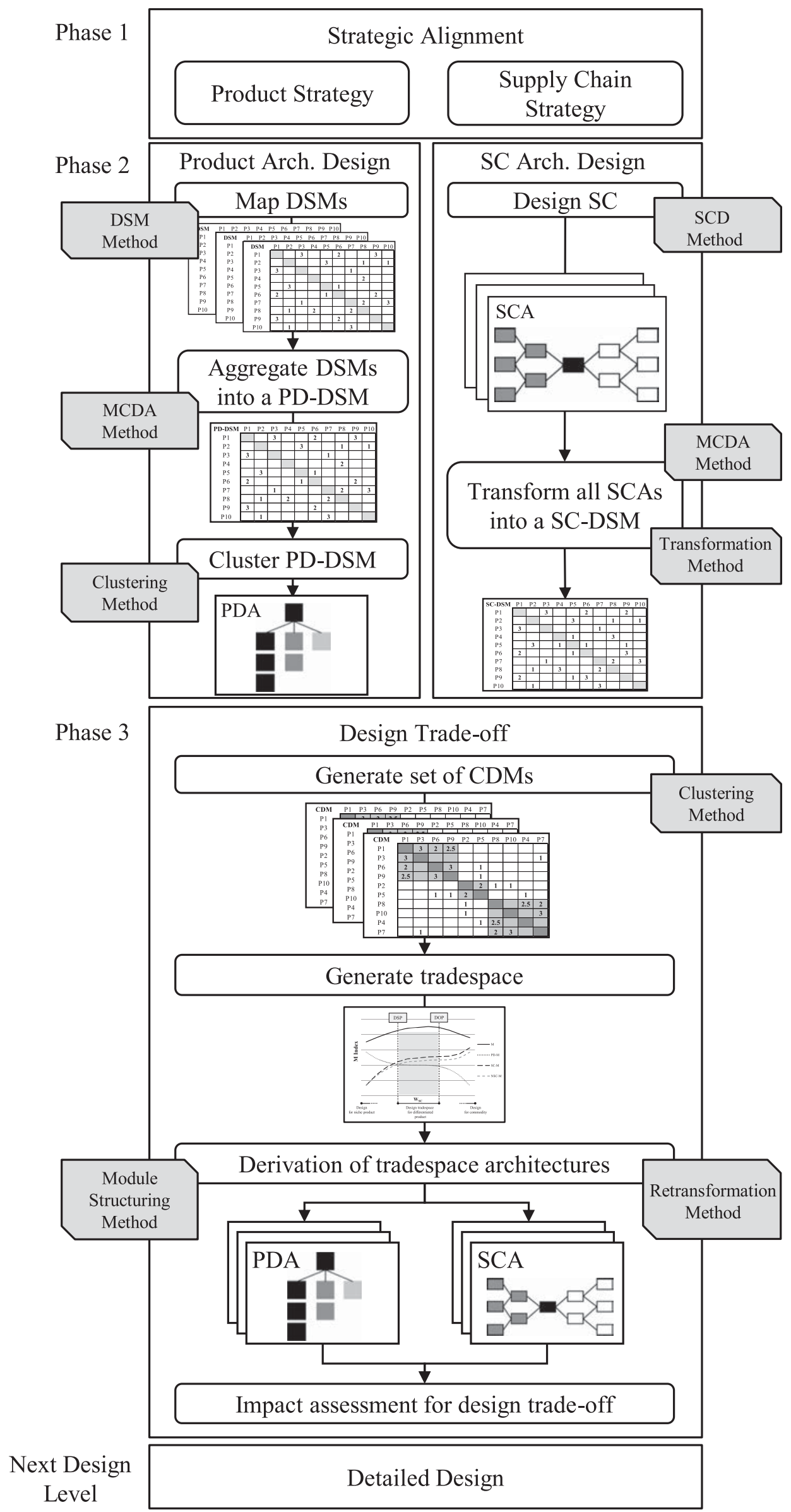

Figure 2. Overview of the CP-SCD process. 
In Phase 3, the PD-DSM and the SC-DSM are normalised, weighted, and aggregated into a single DSM which is then clustered to form the Clustered Decision Matrix (CDM). The CDM represents the architecture of the respective P-SC system at different points in the design tradespace. By varying the relative weights between the PD-DSM and the SC-DSM, CDMs are generated and used to compute the corresponding PD-M, SC-M and $M$ values. These values are used to plot the three curves to represent the design tradespace of the P-SC system. For an in-depth analysis of every selected $\mathrm{CDM}$, the corresponding PDA and SCA are generated for impact assessment. While the PDA is derived directly from the CDM, the SCA has to be retransformed from the CDM using a specific SCD retransformation method. The PDAs and SCAs are assessed according to specific criteria before the transition to the next step for detailed design.

The fundamental contributions to the practice of CPSCD resulting from our methodology lie in the structuring of the concurrent design interactions, design tradeoffs, as well as discussions between the product designers and the SC designers in the design process. It shows in a novel hierarchical way how PD and SCD strategies are defined and linked to a symmetrical design trade-off at architectural-strategic level using modularity as the common design attribute. As Phase 1 is elaborated in Gan and Grunow (2016), we focus on Phase 2 and Phase 3 in the following.

\subsection{Product architectural design}

\subsubsection{General procedure}

The product architectural design stream of Phase 2 involves mapping of DSMs, aggregation of DSMs into a PD-DSM, and clustering of the PD-DSM to create the PDA.

The first step maps the interactions of the PD attributes (e.g. electrical, structural, thermal, spatial, signal, compatibility, lifecycle factors) between product parts in a set of numerical DSMs. We use a numerical DSM method because of its higher information content as compared to a binary DSM method. The upper righthand side of the DSM indicates feed-back interaction and the lower left-hand side of the DSM indicates feedforward interaction. Before mapping PD attributes in the DSM, the product needs to be broken down into parts. The granularity of the parts depends on the required depth of analysis and the complexity of the product. The PD-DSM of the case study is shown in Figure 3.

The second step aggregates DSMs into a single DSM (PD-DSM). In order to map all functional and relational interactions between the parts, the DSM of each PD attribute is weighted and aggregated. The weights of the PD attributes can be determined by any MCDA method that elicits the relative importance of the design attributes from all CP-SCD process stakeholders (e.g. Analytical Hierarchical Process or PROMETHEE).

The third step clusters the PD-DSM to form modules which define the PDA. Clustering methods either use a predefined number of clusters or determine the number of clusters as a part of the method (e.g. Kusiak and Chow 1987; Helmer, Yassine, and Meier 2010; Jung and Simpson 2017). Figure 3 shows the results at the end of Phase 2.

\subsubsection{Specific clustering method for modularisation}

One of the key features of the product architectural design procedure is its modular interface to the clustering method for the identification of modules. This allows a flexible use of different clustering methods to cater to different CP-SCD situations, which may have different clustering objectives and constraints. It also allows for the use of new clustering methods that may be developed in the future.

Existing clustering methods employed for DSMs are found to be inadequate for our case study due to their lack of control over the number and size of identified modules. These are important to the CP-SCD process as the number and size of the modules are key constraints for the PDA (e.g. design teams' workshares) and the SCA (e.g. number of potential suppliers). This motivates us to develop a new heuristic clustering method.

There are two advantages of this new method with respect to design-thinking. Firstly, it uses seed parts in the product modularisation process. Using a search algorithm in this method, designers can identify and select seed parts that are best suited for clustering other parts to form modules (e.g. key interfacing components like product housing and electrical wiring). Secondly, it utilises the designers' knowledge by allowing them to predefine the number and size of modules. The number of predefined clusters often has limited possible values which are determined by internal and external constraints such as organisation, production locations, and manufacturing processes. If required, the method can also be applied for different parametrisations.

Jung and Simpson (2017) introduce the use of interaction strength and number as part of a mathematical approach to cluster DSMs. Our method differs from theirs in the following ways. Firstly, our method is based on the identification of seed parts using interaction numbers for weighting the interaction strengths. The choice of seed parts depends on their strength and number of interactions with other parts. Secondly, we propose a heuristic 


\begin{tabular}{|c|c|c|c|c|c|c|c|c|c|c|c|c|c|c|}
\hline PD-DSM & P1 & P2 & P3 & P4 & P5 & P6 & P7 & P8 & P9 & P10 & P11 & P12 & P13 & P14 \\
\hline P1 & & 1.17 & 0.66 & 0.68 & 1.17 & 0.02 & & & & 0.28 & 0.02 & 0.04 & 0.56 & 0.60 \\
\hline P2 & 1.17 & & 1.57 & 1.33 & 0.10 & & 0.24 & 0.24 & 0.24 & 0.26 & 0.24 & & 0.02 & \\
\hline P3 & 0.66 & 1.57 & & 1.77 & 0.10 & & 1.32 & & 1.10 & & 0.44 & & 0.66 & \\
\hline P4 & 0.68 & 1.33 & 1.77 & & 0.10 & & 0.24 & 0.24 & 0.24 & 1.47 & 0.47 & & 0.02 & \\
\hline P5 & 1.17 & 0.10 & 0.10 & 0.10 & & 1.09 & & & & & & 0.02 & & 0.56 \\
\hline P6 & 0.02 & & & & 1.09 & & 0.10 & 0.10 & 0.10 & 0.38 & 0.66 & 0.98 & 0.10 & 0.96 \\
\hline P7 & & 0.24 & 1.32 & 0.24 & & 0.10 & & 0.84 & 0.86 & 0.56 & 0.62 & 0.94 & 0.12 & 0.10 \\
\hline P8 & & 0.24 & & 0.24 & & 0.10 & 0.84 & & 0.84 & 0.56 & 0.62 & 0.94 & 0.12 & 0.10 \\
\hline P9 & & 0.24 & 1.10 & 0.24 & & 0.10 & 0.86 & 0.84 & & 0.56 & 0.62 & 0.94 & 0.12 & 0.10 \\
\hline P10 & 0.28 & 0.26 & & 1.47 & & 0.38 & 0.56 & 0.56 & 0.56 & & 1.18 & 0.59 & 0.84 & 0.10 \\
\hline P11 & 0.02 & 0.24 & 0.44 & 0.47 & & 0.66 & 0.62 & 0.62 & 0.62 & 1.18 & & 0.96 & 0.32 & 0.10 \\
\hline P12 & 0.04 & & & & 0.02 & 0.98 & 0.94 & 0.94 & 0.94 & 0.59 & 0.96 & & 0.12 & 0.12 \\
\hline P13 & 0.56 & 0.02 & 0.66 & 0.02 & & 0.10 & 0.12 & 0.12 & 0.12 & 0.84 & 0.32 & 0.12 & & 0.68 \\
\hline P14 & 0.60 & & & & 0.56 & 0.96 & 0.10 & 0.10 & 0.10 & 0.10 & 0.10 & 0.12 & 0.68 & \\
\hline
\end{tabular}

\begin{tabular}{|l|l|l|l|l|}
\hline \multicolumn{1}{|c|}{ PDA } & M1 & M2 & M3 & M4 \\
\hline P1 & & & & \\
\hline P5 & & & & \\
\hline P6 & & & & \\
\hline P11 & & & & \\
\hline P13 & & & & \\
\hline P14 & & & & \\
\hline P2 & & & & \\
\hline P3 & & & & \\
\hline P4 & & & & \\
\hline P7 & & & & \\
\hline P8 & & & & \\
\hline P9 & & & & \\
\hline P10 & & & & \\
\hline P12 & & & & \\
\hline
\end{tabular}

Definition of rating scheme

Empty - No interaction

1 - Weak functional / relational interaction

2 - Medium functional / relational interaction

3 - Strong functional / relational interaction

Figure 3. Phase 2 result showing the PD-DSM and the PDA.

algorithm that facilitates the integration of additional design knowledge.

Heuristic algorithm. Seed parts are identified in the PD-DSM by an algorithm using two criteria. Firstly, a seed part $i$ shall have a high number of interactions $c_{i j}$ and high interaction strengths $r_{i j}$ with other parts $j$ (Figure 4). To ensure that both the number of interactions and the interaction strength are considered, the algorithm starts the search from the part $i$ with the highest value for the seeding function $W S_{i}$, defined as the product of both values:

$$
W S_{i}=\sum_{j \in J} c_{i j} \sum_{j \in J} r_{i j}
$$

where:

$r_{i j}$ Interaction strength between part $i$ and part $j ; c_{i j}$ Binary parameter indicating an interaction between part $i$ and part $j\left(c_{i j}=1\right.$, iff $\left.r_{i j}>0\right)$.

Secondly, a seed part shall have low interaction strengths with other seed parts. For the identification of seed parts, the algorithm uses a filter (F-level) to differentiate weak interactions from strong interactions.

The procedure of the heuristic algorithm is shown in Figure 5 and pseudocode is included in Appendix A1, see supplemental data. Firstly, the target number of seed parts and the initial $F$-level are set (default value $=0$ ). This is followed by conversion of the DSM to a Filtered Matrix (Figure 4). This Filtered Matrix decouples the

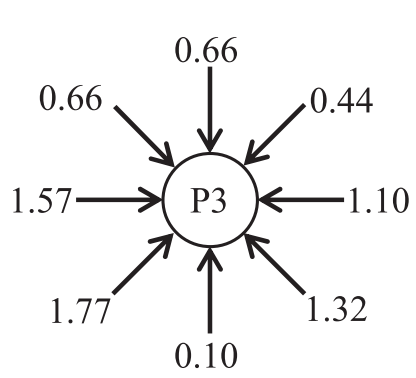

$$
\begin{aligned}
& \sum_{j} r_{3 j}=7.62 \\
& \sum_{j} c_{3 j}=8
\end{aligned}
$$

Identified seed parts in the Filtered Matrix at F-level $=0.13(\mathrm{P} 11, \mathrm{P} 2, \mathrm{P} 13, \mathrm{P} 5)$

Figure 4. The Filtered Matrix for an F-level of 0.13 (case study data). 


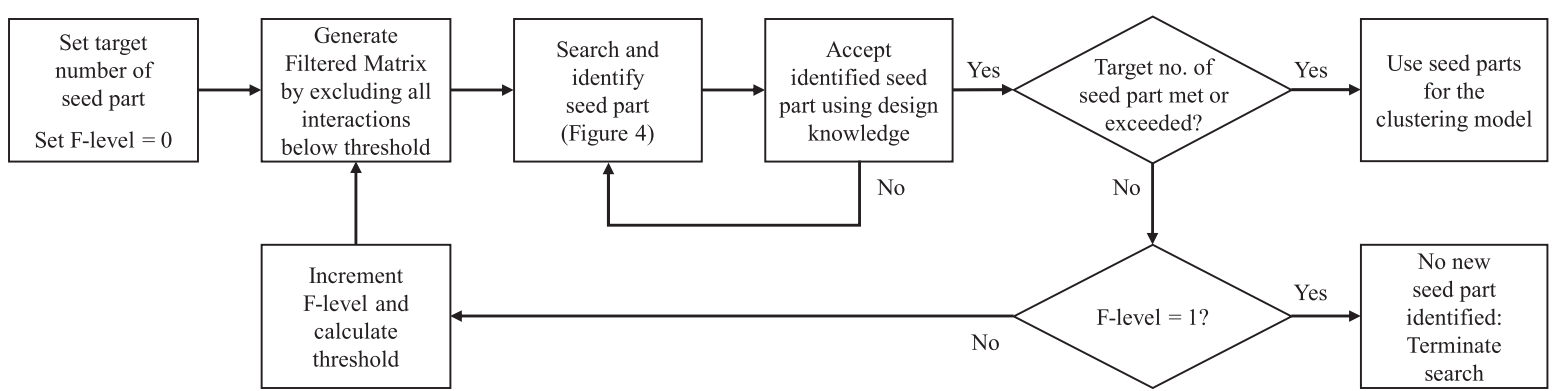

Figure 5. Procedure of the heuristic algorithm for the selection of seed parts.

parts by suppressing DSM interactions that are below the F-value, with $F$-value $=F$-level $\cdot \max _{i, j} r_{i, j}$.

In the next step, the parts in the Filtered Matrix are sorted in descending order according to their $W S_{i}$ values. Next, the part with the highest $W S_{i}$ value is assigned as the first seed part. The algorithm then searches downwards, row-by-row, for the next seed part. A part is identified as a seed part if it is not coupled with the preceding seed parts (e.g. P11 $\rightarrow \mathrm{P} 2 \rightarrow \mathrm{P} 13 \rightarrow \mathrm{P} 5$ in Figure 4). However, if any of these parts should not be chosen based on additional design knowledge, they can be skipped. This additional knowledge is used to determine the suitability of the part as seed parts due to certain physical (e.g. spatial location) and non-functional attributes (e.g. value) that are not captured in the DSM. For instance, a low-value commodity part would not be chosen as a seed part. This algorithm terminates once the target number of seed parts has been reached or if the F-level has reached the value of one. Otherwise, the F-level is incremented, and the abovementioned steps are repeated.

After the identification of seed parts, a quadratic integer programming model is used to determine the modules. It maximises the interactions between the identified seed parts and the non-seed parts assigned to them, while ensuring the number and size constraints of the modules.

Sets:

$J$ Set of all parts $j$

$I$ Set of seed parts $i(I \subseteq J)$

\section{Parameters:}

$n$ Minimum size of a cluster

$N$ Maximum size of a cluster

\section{Decision variables:}

$$
x_{i j}= \begin{cases}1, & \text { part } j \text { is assigned to seed part } i \\ 0, & \text { part } j \text { is not assigned to seed part } i\end{cases}
$$

Module formation model:

$$
\operatorname{Max}\left(\sum_{i \in I} \sum_{j \in J I} x_{i j} r_{i j}+\frac{1}{2} \sum_{i \in I} \sum_{j \in J I} \sum_{k \in J \backslash I, k \neq j} x_{i j} x_{i k} r_{j k}\right)
$$

Subject to:

$$
\begin{array}{ll}
\sum_{i \in I} x_{i j}=1 & \forall j \in J \backslash I \\
n-1 \leq \sum_{j \in J \backslash I} x_{i j} \leq N-1 & \forall i \in I
\end{array}
$$

The objective function (2) maximises the interactions between the seed parts $i$ and the non-seed parts $j$ (first expression) as well as the interactions between all nonseed parts $j$ and $k$ in all clusters (second expression). Constraints (3) ensure that each part $j$ is assigned to exactly one seed part $i$. Constraints (4) ensure minimum and maximum cluster sizes. The quadratic assignment problem (Equations (2-4)) can be solved by a non-linear solver such as a quadratic and general reduced gradient solver or a meta-heuristic such as a genetic algorithm depending on the size of model. For the case study (Figure 4), we set the target number of seed parts as four and the minimum cluster size as two. The seed parts are determined at $F$-level $=0.13$. The four resulting modules consist of the seed parts (P2, P5, P11 and P13) and their assigned parts (Figures 3 and 4). Using a laptop with Intel ${ }^{\circledR}$ Core i7 processor and 16GB RAM, the mode of the clustering method is solved by the genetic algorithm in Frontline Solver Pro (60 sec).

\subsection{Supply chain architectural design}

\subsubsection{General procedure}

The general procedure for the SC architectural design stream of Phase 2 comprises an SCD step and a transformation step which generate SCAs and convert the SCAs into an SC-DSM respectively. In comparison to PD attributes mapped in a part-to-part DSM, SCD may use other attributes (e.g. suppliers, delivery lead times, location, or manufacturing technologies) to consider certain SC objectives (e.g. costs, speed, or sustainability). An SC-DSM also needs to map parts to parts to be able to link the PD and SCD domains. Hence, an SCA has to be 
transformed into an SC-DSM. For cases that involve multiple SCAs, this transformation includes a weighting and aggregation procedure based on a MCDA methodology.

As in the case of the PD stream, a key characteristic of the SCD stream is its capability to use different SCD methods. While there are numerous SCD methods, transformation methods to convert the resulting SCAs into representations that are commensurable with PDAs have not been developed.

SC architectures can be designed with many different objectives. In the next section, we introduce a specific optimisation-based method for generating the SCA when aiming at an efficient supply base with a minimum number of suppliers and supply modules that still allow for flexible sourcing. In general, the SC architecture could however be constructed with different objectives in mind, and with different methods. For instance, the SCD could involve typical location-allocation decisions on what parts to assemble at which locations. These decisions can be supported in many ways, ranging from simple scenario analyses to optimisation models that quantify expected production, transportation, and inventory costs in detail. Such models could also include richer information such as the restriction of the allocation of parts based on sensitive intellectual property to certain locations.

For the process we propose in this paper, the only requirement is that the resulting SCA can be translated into a DSM, so that it can be consolidated with the results from the concurrently designed product architecture in Phase 3. For the above-mentioned location-allocation example, the parts being assembled at the same location would for instance receive positive entries in the SC-DSM.

\subsubsection{Specific method for sourcing flexibility}

Our SCD method allocates parts to supply modules that are assigned to suppliers. We define sourcing flexibility as the number of suppliers assigned to a supply module. The supply scope of a supplier is defined by the parts that it can supply. It is represented in a Part-Supplier Matrix (PSM). Each '1' in the PSM indicates that the part (row) can be sourced from the supplier (column) (cf. Appendix A2, see supplemental data). Single-sourced parts are not considered in the PSM as they offer no sourcing flexibility to the SC design space. The creation of the PSM considers various determinants for supplier selection by using MCDA methods (Ho, Xu, and Dey 2010). Suppliers that do not meet those determinants, such as quality and performance, are not considered in the PSM. A bi-objective binary integer programming is used to first minimise the number of suppliers and then the number of supply modules using a lexicographic approach, while ensuring the required sourcing flexibility. We are using the following notation and model formulation:

\section{Parameters:}

$h$ Sourcing flexibility constant

$k$ Upper limit to the number of modules that can be assigned to a supplier

$l$ Upper limit to the number of parts in a module

$q_{p s}= \begin{cases}1, & \text { Part } p \text { can be sourced from the supplier } s \text { in the PSM } \\ 0, & \text { Part } p \text { cannot be sourced from the supplier } s \text { in the PSM }\end{cases}$

Decision variables:

$$
\begin{aligned}
y_{m s} & = \begin{cases}1, & \text { Module } m \text { is assigned to a supplier } s \\
0, & \text { Module } m \text { is not assigned a supplier } s\end{cases} \\
x_{p m} & = \begin{cases}1, & \text { Part } p \text { is assigned to a module } m \\
0, & \text { Part } p \text { is not assigned a module } m\end{cases} \\
t_{s} & = \begin{cases}1, & \text { Supplier } s \text { is selected } \\
0, & \text { Supplier } s \text { is not selected }\end{cases} \\
c_{m} & = \begin{cases}1, & \text { Module } m \text { is selected } \\
0, & \text { Module } m \text { is not selected }\end{cases}
\end{aligned}
$$

Sourcing model:

$$
\begin{aligned}
& \operatorname{Min} \sum_{s \in S} t_{s} \\
& \operatorname{Min} \sum_{m \in M} c_{m}
\end{aligned}
$$

Subject to:

$$
\begin{array}{ll}
\sum_{m \in M} x_{p m}=1 & \forall p \in P \\
x_{p m} \leq c_{m} & \forall p \in P, \forall m \in M \\
y_{m s} \leq t_{s} & \forall m \in M, \forall s \in S \\
\sum_{p \in P} x_{p m} \geq y_{m s} & \forall m \in M, \forall s \in S \\
c_{m+1} \leq c_{m} & \forall m \in M \backslash\{|M|\} \\
\sum_{p \in P} p \cdot x_{p m} \geq \sum_{p \in P} p \cdot x_{p m+1} & \forall m \in M \backslash\{|M|\} \\
\sum_{s \in S} y_{m s} \geq h \cdot c_{m} & \forall m \in M \\
\sum_{m \in M} y_{m s} \leq k & \forall s \in S \\
\sum_{p \in P} x_{p m} \leq l & \forall m \in M
\end{array}
$$




$$
\begin{array}{ll}
\sum_{s \in S^{r}} y_{m s} \geq 1 & \forall m \in M \\
x_{p m}+y_{m s} \leq q_{p s}+1 & \forall p \in P, \forall m \in M, \forall s \in S
\end{array}
$$

The first objective function (5) minimises the number of suppliers $t_{s}$ and the second objective function (6) minimises the number of supply modules $c_{m}$ to improve the efficiency of the SCD. Constraints (7) ensure that each part is assigned to a single module. Constraints (8) ensure that no part is assigned to a module if the module is not used. Constraints (9) ensure that no module is assigned to a supplier if the supplier is not selected. Constraints (10) ensure that no supplier is assigned to a module if the module has no assigned parts. Constraints (11) and (12) are symmetry-breaking constraints. Constraints (13) ensure the required sourcing flexibility, which is defined by the constant $h(h=2$ for double sourcing; $h=3$ for triple sourcing, etc.). Optionally, constraints (14)-(16) can be applied. To limit the suppliers' power over the OEM, constraints (14) ensure the maximum number of modules that can be assigned to the suppliers. Constraints (15) ensure the maximum size of the supply modules. Constraints (16) ensure that there is at least one responsive supplier in each supply module. Finally, constraints (17) ensure the feasibility of the result with respect to the PSM.

The output of this step defines the optimal assignment of the parts to the supply modules $\left[x_{p m}\right]$ and the supply modules to the supplier groups with flexible sourcing $\left[y_{m s}\right]$. The result of the transformed SCA with double sourcing flexibility using data from the case study is shown in Figure 6. It is important to note that it is possible that the coupling of the supplier groups (e.g. S4 in Figure 6) can occur. This occurs if the PSM, which is the feasibility constraint of the clustering model, does not have sufficient flexibility to allow for completely disjunct supplier groups. Using the computational specification, the model of the SCD method is solved by CPLEX $(0.1 \mathrm{sec})$.
The transformation method for the conversion of the SCA into an SC-DSM uses the sizes of the supply modules to identify the interaction strength between parts (Figure 6). The number of parts assigned to each supply module can be identified in the SCA. Fewer and bigger modules are beneficial due to lower transaction costs (e.g. managing suppliers) and final assembly lead time.

\subsection{Design trade-off}

Phase 3 of the CP-SCD process involves the generation of the set of CDMs and the M curve, the identification of the design tradespace, the derivation of design architectures, and the impact assessment for design trade-off.

\subsubsection{Generation of the CDMs}

The generation of the set of CDMs is based on the normalised PD-DSM and SC-DSM. The left side of Figure 7 shows the normalisation of the SC-DSM using the scale from the PD-DSM. This ensures that the PD-DSM and the normalised SC-DSM have interaction values that are on the same scale, thereby ensuring their aggregability.

First, the interactions of the two DSMs are aggregated into a single DSM (C-DSM, top right of Figure 7), making the PDA and the SCA commensurable. Next, the C-DSM is clustered to generate the CDM, which represents the modules obtained from an integrated product and SC perspective. Any clustering method may be used, including the method proposed in Section 4.2.2. For the clustering of the PD-DSM and the C-DSM, the design team predefines a target of four modules, while keeping the number of supply modules and supplier groups in SCA unrestricted. Even though the number of supply modules identified in the SCA and the number of modules in the PDA are identical, the results are independent as they are clustered using different methods. The detailed results are found in Appendix A4, see supplemental data.

To vary the relative importance of both domains, we introduce relative weights $W_{P D}$ and $W_{S C}$, with $W_{P D}=$ $1-W_{S C}$. These weights range from 0 to 1 and have the
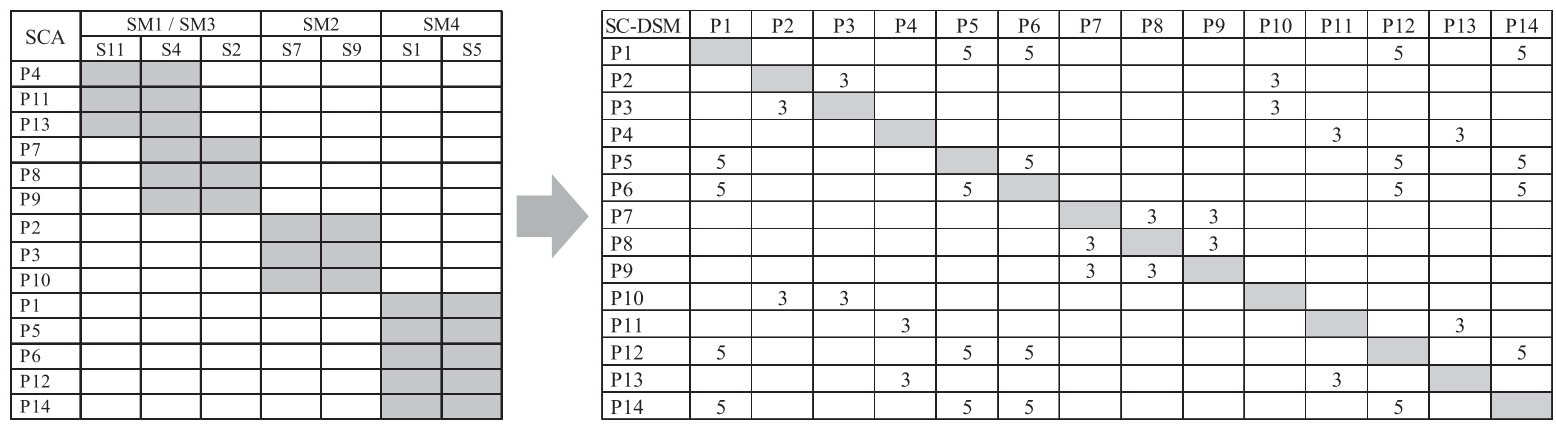

Figure 6. Phase 2 result showing the SCA defined by the Supply Modules (SM) and its transformation to an SC-DSM. 


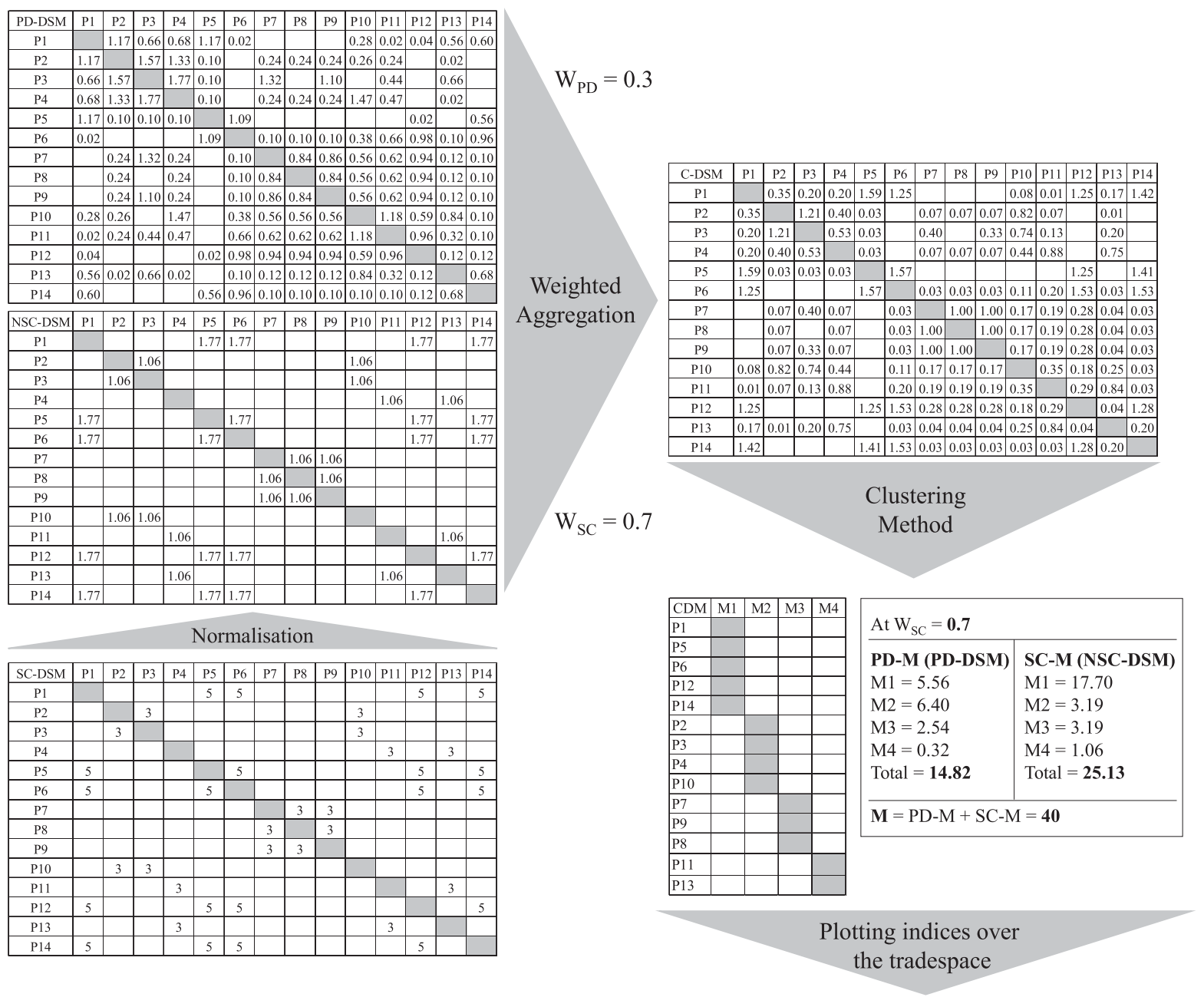

Figure 7. DSM operations for the generation of tradespace.

following design implications: if $W_{S C}$ equals 0 , the P-SC system is exclusively product-centric and if $W_{S C}$ equals 1 , the P-SC system is exclusively SC-centric. Varying weights avoids the need to predetermine the values of the weights. Instead, this typical MCDA approach generates and explores the design tradespace.

\subsubsection{Generation of the tradespace}

The CDM modules are assessed from the product perspective as well as the SC perspective by referring to the original DSMs. The following indices quantify the modularity level of the PDA and the SCA for the modules represented in the CDM:

- $P D-M$ index: Non-weighted sum of PD-DSM interactions captured by the CDM modules

- SC-M index: Non-weighted sum of SC-DSM interactions captured by the CDM modules

- NSC-M index: normalised SC-M index

- $M$ index: Sum of the $P D-M$ and $S C-M$ values
The modularity indices can subsequently be plotted over the entire range of $W_{S C}$ to characterise the design tradespace of the P-SC system. The resulting PD-M, SC$M, N S C-M$, and $M$ curves presented in Figure 8 show the modularity levels of the PD, the SCD and the P-SC system respectively.

Here it can be seen that the potential designs for weights $W_{S C}=0.2$ and $W_{S C}=0.3$ are identical. The same holds true for the designs for weights $W_{S C} \geq 0.8$. In total, eight different modules in the CDM were detected. If weights were varied at smaller intervals, even more potential designs might be identified. The PD-M curve has a generally negative gradient, and the SC-M curve has a generally positive gradient. The modularity curves thus illustrate a trade-off. The exception are cases in which the same modules are optimal from a product perspective as well as a supply chain perspective.

Gan and Grunow (2016) define the Design Optimum Point (DOP) as the point in the tradespace where the design attribute of a P-SC system is the highest. Graphically, the DOP is located at the apex of the M curve. 


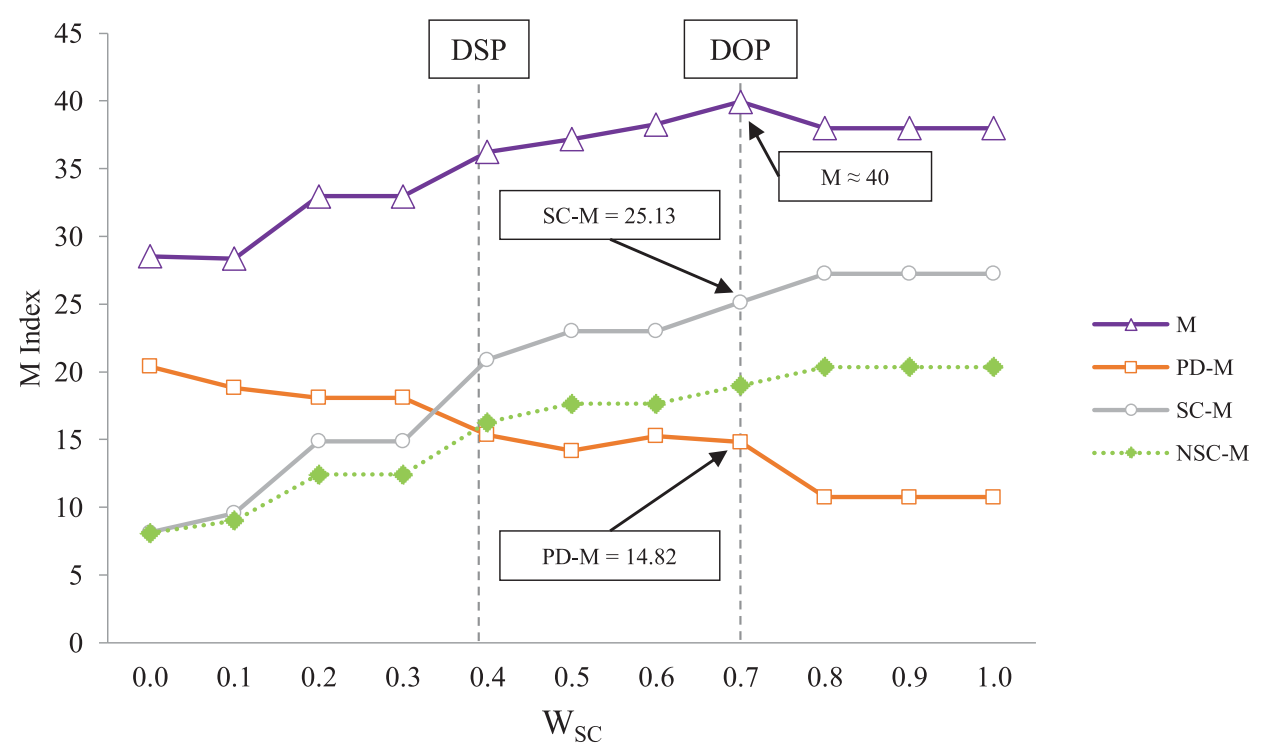

Figure 8. Representation of the tradespace by the PD-M, SC-M, NSC-M and M curves.

This concept can be applied to all commensurable design attributes. The bottom right of Figure 7 shows the three indices at $W_{S C}$ equals 0.7 (DOP). Here, we obtain values of $\mathrm{PD}-\mathrm{M}=14.82$, SC-M $=25.13$ and $\mathrm{M} \approx 40$.

The DOP by itself does not provide sufficient information to conduct a design trade-off. In this paper, we therefore introduce the Design Symmetry Point (DSP), defined as the point in the tradespace where the normalised values of the PD and the SCD attributes are identical (with normalisation related to their maximum values). Hence, the DSP reflects an equitable trade-off point. It is important to note that the DOP and the DSP are always specific to the scope of design trade-off and that the DSP can be positioned on either side of the DOP, depending on the P-SC system. Together, these points help characterise the tradespace and enhance the trade-off process in the following ways:

(1) Quantification of the alignment level between the $\mathrm{PD}$ and the SCD as an endogenous characteristic of the P-SC system.

(2) Demarcation of different areas of the tradespace relevant for different types of products.

(3) Enrichment of design trade-off process using visual graphs.

For niche products and commodities, equitable consideration of both design domains is not necessary since one of the domains dominates the design process. For niche products, the part of the tradespace near the solution with exclusive consideration of $\mathrm{PD}$ requirements is relevant. Similarly, for commodities, the part of the tradespace near the solution with exclusive consideration of SCD requirements is relevant.

For differentiated products such as the battery system investigated in our case study, the DSP and the DOP provide relevant insights on the characteristics of the tradespace. The larger the distance between the DSP and the DOP, the larger the effort required for the design trade-off. Exploring the design space between the DOP and the DSP, we balance the optimality of the entire P-SC system with the equitable consideration of both domains. At every exploration step, feasibility studies are conducted to assess the impact of the designs. In Figure 8, the DOP is located to the right of the DSP. This indicates a higher P-SC system modularity when the P-SC system architecture is oriented towards the SCD. Between the DSP and the DOP is a gap of 0.3 in the tradespace, representing the tradespace between equitability and optimality in the P-SC system. In order to assess the impacts of these two points on the P-SC system architecture, we present the comparisons of their respective CDMs $\left(\mathrm{CDM}_{\mathrm{DSP}}\right.$ and $\left.\mathrm{CDM}_{\mathrm{DOP}}\right)$ with the $\mathrm{CDM}_{0}\left(W_{S C}=0\right)$ and the $\mathrm{CDM}_{1}\left(W_{S C}=1\right)$ in the following section.

For the designers in the case study, the generated tradespace (Figure 8) offered a concrete view of the design trade-off. This visual representation of the tradespace provided a common understanding of the $\mathrm{P}$ SC system. By starting with the DSP and the DOP, the designers were quickly able to evaluate important points in the tradespace to be studied further in the impact assessment. We have not found any previous study that provides a method of generating a tradespace in CP-SCD. 


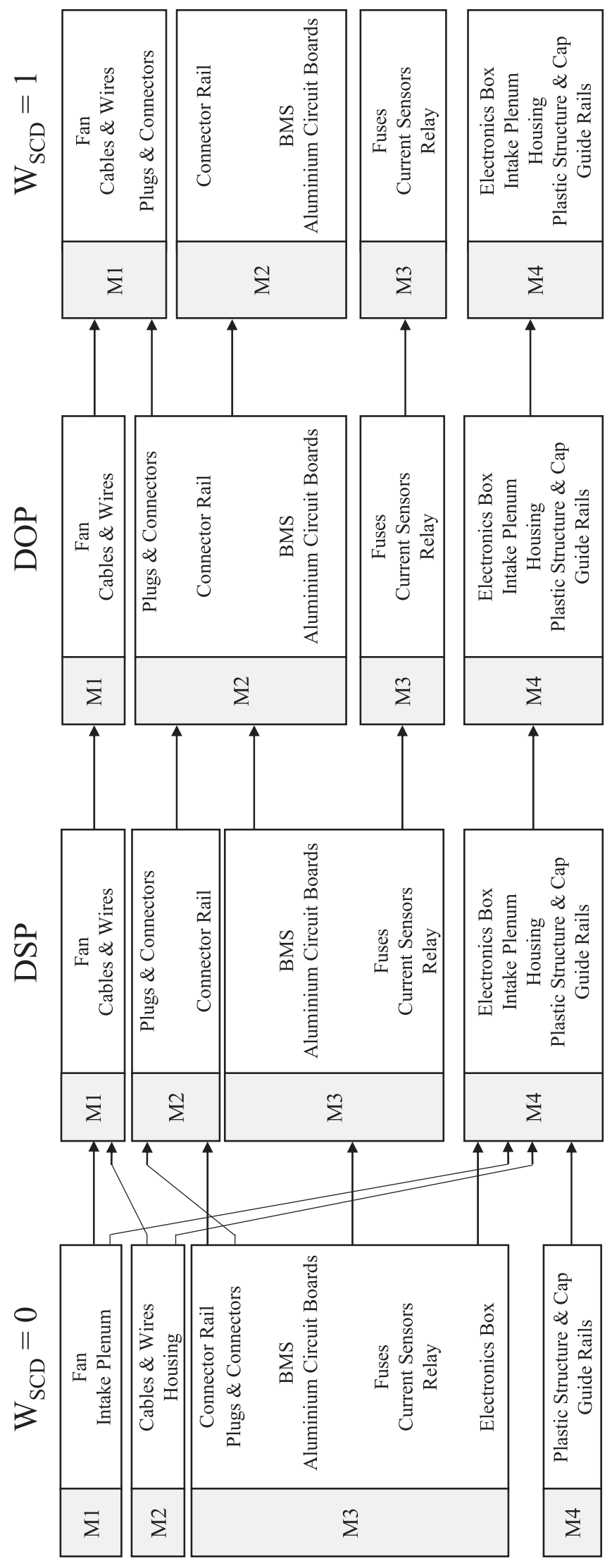

Figure 9. Impact assessment of the architectures at the DSP and the DOP. 


\subsubsection{Derivation of tradespace architectures}

The next step in Phase 3 includes the derivation of PDAs and SCAs. The derivation of SCAs requires an additional step to retransform the selected CDMs into the corresponding SCA. The retransformation of the CDM to the SCA is needed to ensure the optimal grouping of the suppliers, which may differ from the supplier groups of the SCA derived from Phase 2. The retransformation method is a modification of the SCD method. The modification includes a new objective function (18) for maximising the similarity between the part-to-supplier group assignment $x_{p m}$ of the SCA and the part-to-module assignment $u_{p m}$ of the CDM. Constraints (12) are omitted to ensure the feasibility of the solutions.

$u_{p m}= \begin{cases}1, & \text { Part } p \text { is assigned to the module } m \text { in the CDM } \\ 0, & \text { Part } p \text { is not assigned to the module } m \text { in the CDM }\end{cases}$

(a)

PDA (DSP)

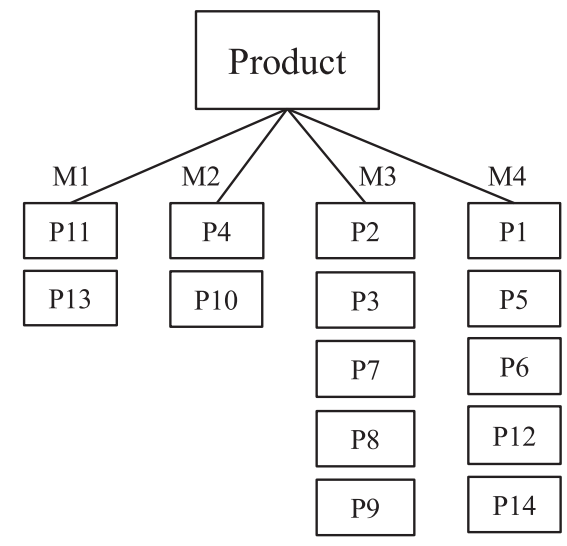

(c)

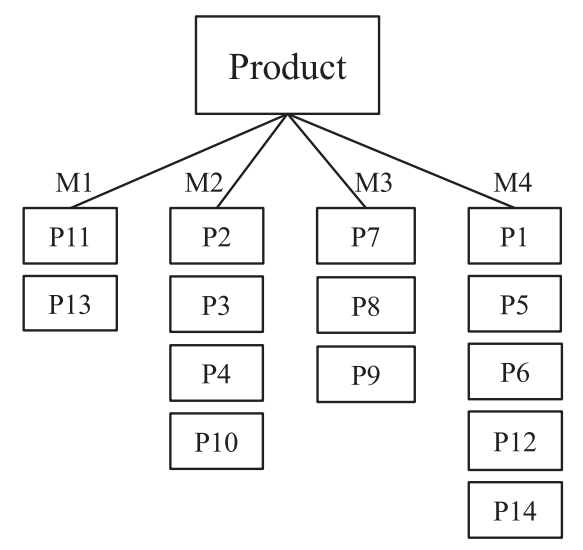

$\operatorname{Max} \sum_{p \in P} \sum_{m \in M} x_{p m} u_{p m}$

\subsubsection{Impact assessment for design trade-off}

In the final step of Phase 3 of the CP-SCD process, the impact assessment focuses on the space between DSP and DOP. In our case study, this facilitated a discussion on the tangible design options constituting the tradespace. In the example presented in Figure 9, we compare the modules found for the DOP and the DSP with each other. For illustration purposes, we also contrast them against the modules generated for $\mathrm{CDM}_{0}$ and $\mathrm{CDM}_{1}$. These comparisons highlight the differences in the size and the content of the modules.

The differences between the CDMs and the $\mathrm{CDM}_{0}$ are assessed directly with regards to product design impacts such as technical feasibility (e.g. functional degradation,
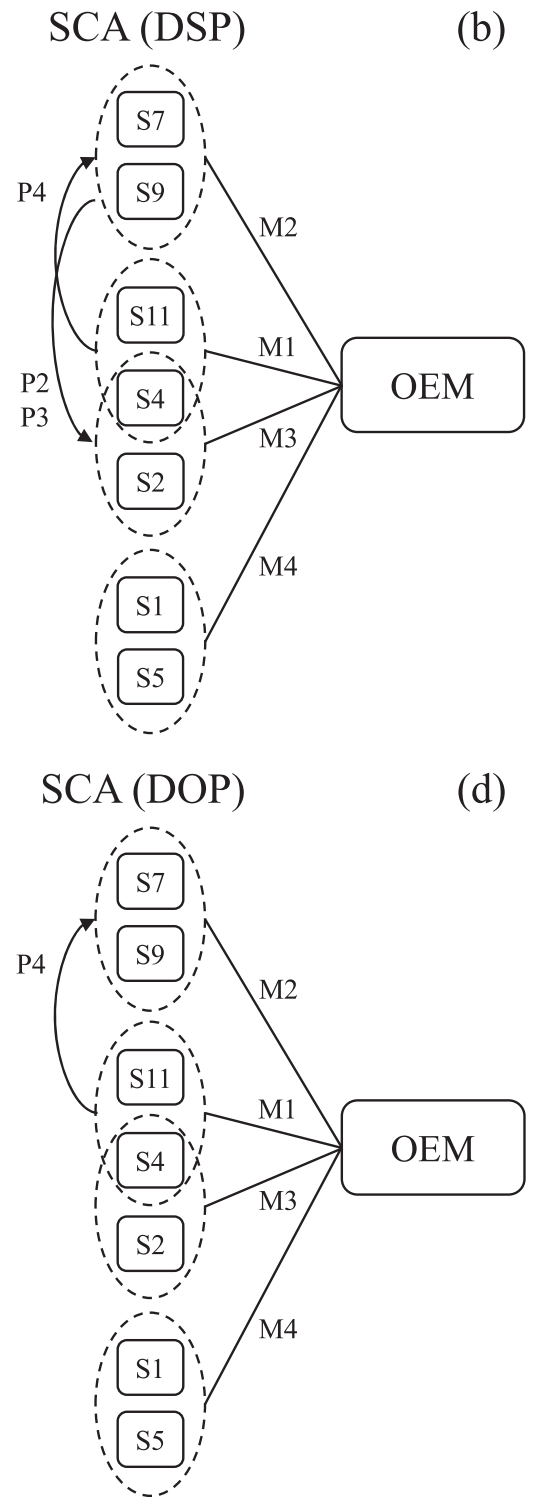

Figure 10. Hierarchy diagrams of PDA (a) and SCA (b) at DSP, and PDA (c) and SCA (d) at DOP. 
weight, space). For example, the architecture of the $\mathrm{CDM}_{D S P}$ has a major impact on the functional integrity of the $\mathrm{CDM}_{0}$ modules. The $\mathrm{CDM}_{0}$ modules M1, M2 and M3 are disintegrated and distributed over all modules of the $\mathrm{CDM}_{D S P}$. Only the $\mathrm{CDM}_{0}$ module M4 remains integral. Major impacts of the $\mathrm{CDM}_{D S P}$ on the $\mathrm{CDM}_{1}$ can similarly be observed as only the $\mathrm{CDM}_{1}$ module $\mathrm{M} 4$ remains intact.

In comparison to the $\mathrm{CDM}_{D S P}$, the $\mathrm{CDM}_{D O P}$ has similar impact on the $\mathrm{CDM}_{0}$ and only a minor impact on the $\mathrm{CDM}_{1}$. The $\mathrm{CDM}_{0}$ module $\mathrm{M} 3$ is redistributed to three of the $\mathrm{CDM}_{D O P}$ modules, as in the case of the $\mathrm{CDM}_{D S P}$. The $\mathrm{CDM}_{1}$ modules $\mathrm{M} 3$ and $\mathrm{M} 4$ remain intact. M2 is almost unchanged except for the plugs \& connectors, which are added from the M2. Another result of the impact assessment relates to the change of the SCA complexity. We highlight this result by focussing on the material flows within the SCA. These changes affect how the parts can be sourced (i.e. part, supply kit or module). A module is a set of assembled parts that can be further assembled. A supply kit is a set of parts delivered by the same supplier without any functional association (e.g. a box of loose parts). As such, the use of supplier type in the SCA has a different impact on the lead time, assembly and testing in production.

The increase in SC complexity is indicated by the flows of parts between the SCA modules at the DSP and the DOP shown in the hierarchy diagrams (Figure $10(\mathrm{~b}, \mathrm{~d})$ ). For the SCA at the DSP, the supplier group S4/S11 changes from a module supplier group to a mixed module and part supplier group, which delivers the part P4 to S7/S9 (Figure 10(b)).

Furthermore, the supplier group S7/S9 changes from a module supplier group to a mixed module and kit supplier group, which delivers the kit (P2/P3) to S2/S4. As a result, both supplier groups become first tier and second tier supplier groups. This additional SC layer increases the lead time and the cost of production (e.g. inventory and transportation). In comparison to the SCA at the DSP, the SCA at the DOP is less complex as it only has to additionally source P4 from S4/S11 to S7/S9 (Figure $10(d))$.

Even though sourcing flexibility is ensured in the resulting SCAs, only seven out of the 14 potential suppliers were selected to supply the four modules (Figures 6 and 10). These modules are however very different from the modules that would be created for an optimal PD (as was shown in Figure 9).

Overall, the SCAs at the DSP and the DOP reduce the number of suppliers that are managed directly by Invenox and the associated transaction costs. Comparing the PSC architectures at pure product orientation, DSP, DOP, and pure SC orientation, the designers found that the advantages of a more modular and flexible SCD obtained at DOP outweighed the technical disadvantages for the PD. The impact assessments supported by visualisations such as Figures 9 and 10 thus helped balance between PD and SCD objectives in the case.

\section{Conclusion}

This paper advances research in CP-SCD by linking theory and methodology to practice. Firstly, we built a better understanding of the interaction between PD and SCD by introducing a novel way to characterise the design tradespace between a PDA and an SCA. We introduced a new design term (DSP) and showed how it can be used together with the DOP to demarcate the relevant part of the tradespace. We are thus contributing to the state-ofthe-art, which so far did not allow for a comprehensive exploration of the tradespace.

Secondly, we operationalised the conceptual CP-SCD process by introducing a detailed process for generating the tradespace between a PD and an SCD. The detailed process consists of three phases. The first phase aligns $\mathrm{PD}$ and SCD requirements and defines the relevant design attributes. The second phase consists of parallel processes to generate the PD and the SCD. The third phase generates the design tradespace and conducts impact assessments of the selected PDAs and SCAs. This methodology, consisting of a detailed process and methods, goes beyond past work that only proposes conceptual frameworks that are not sufficiently specific to be applied in practice.

Thirdly, we introduced new methods to generate PD and SCD architectures. The PD method goes beyond current clustering methods by allowing greater control of the number and the size of the modules. Our PD method includes a new heuristic algorithm that uses interaction numbers and interaction strengths to select independent seed parts for clustering the parts into modules. The SCD method differentiates itself from the current SCD methods by clustering parts to their suppliers while ensuring sourcing flexibility. More importantly, our SCD method ensures that the SCA is commensurable with its PDA, thereby facilitating design trade-off. Here, we contribute by using DSM representations for both the PD and SCD. In contrast to previous work, this also allows us to use common methods for PD and SCD and to apply MCDA methodology to bridge both domains.

Finally, we address the lack of cases highlighted in our literature review by demonstrating the application of the CP-SCD process and methods in a real case study in the 
electric-vehicle battery industry. The systematic exploration of the design tradespace in the case study showed how a range of solutions provide a better understanding of the trade-off between PD and SCD objectives.

We also realise that our methodology and case study have several possible limitations and areas for future research. Firstly, the result of the CP-SCD process is dependent on quality and promptness of input data. For example, the functional and SC interactions between components may not be fully known in Phase 1 of the PD process. This is especially true for more complex products. A way to address this issue is to update the data and iterate the process until an adequate level of architectural design maturity has been reached.

Secondly, the case study uses an unconstrained PDA with a green-field SCA. However, for cases such as product improvement, the PDA can be influenced by the PDAs of predecessor products. Similarly, the SCA can also be constrained by brown-field SC factors such as the reuse of existing suppliers. These considerations are not covered in our case study.

Finally, our methodology is applicable to PD and SCD under different objectives; our case study and the specific methods developed focus on two design attributes: modularity and flexibility. These are key attributes at the architectural level. Nonetheless, methods for optimising other design attributes (e.g. assembly locations) under different criteria (e.g. costs, responsiveness, or environmental footprints) can also be explored and developed for use in our CP-SCD process, which has been deliberately developed with open interfaces to other methods.

\section{Disclosure statement}

No potential conflict of interest was reported by the authors.

\section{Notes on contributors}

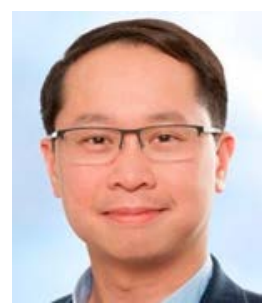

Thiam-Soon Gan is a research associate at the Chair of Production and Supply Chain Management at the Technical University of Munich's TUM School of Management in Germany. He received his BEng degree in Mechanical Engineering from the National University of Singapore and his SM degree in Engineering and Management from the Massachusetts Institute of Technology. He is currently the Head of Program Management at Lilium, a Munich-based aerospace company that designs electrically powered vertical take-off and landing aircrafts. His 17-year industrial career includes various management and technical positions in the automotive, aerospace and defence industries (Airbus SE, Continental AG, and Daimler AG). His research interest focuses on methodology for concurrent design of product, production system and supply chain.

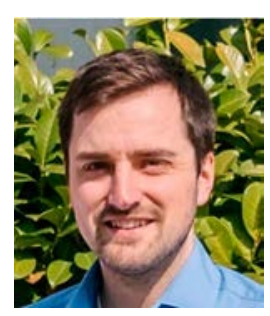

Moritz Steffan is one of the founders of INVENOX, a Munich-based company that designs and produces advanced Lithium-Ion-batteries for mobile machines and systems, and currently works there as the Executive Director of Procurement, Quality and IT. Earlier, he was a research associate at the Institute of Automotive Engineering at the Technical University of Munich. Before that, he received his Diploma (MEng) degree in Automotive Engineering from the Technical University of Munich. He has a special interest in concurrently setting up and managing supply chain, production system and product design for the battery and electronics industries.

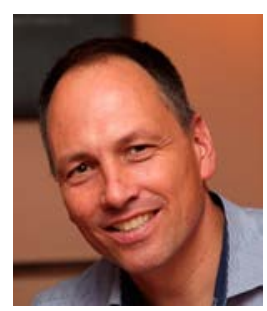

Martin Grunow is a Professor of Production and Supply Chain Management at the Technical University of Munich's TUM School of Management in Germany. He received his MSc degree in Industrial Engineering and his Ph.D. from Technical University of Berlin. After working in the R\&D department of Evonik Degussa, Martin was a Full Professor of Operations Management and Department Head at Technical University of Denmark. In his research, he develops analytics methodology for manufacturing and logistics. Martin has a special interest in the electronics and automotive sector as well as on the process industries, including chemicals, pharmaceuticals, and food.

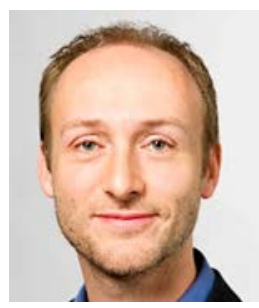

Renzo Akkerman is an Associate Professor in Operations Research and Logistics at Wageningen University in The Netherlands. Earlier, he was a Professor in Operations Management and Technology at the Technical University of Munich in Germany and an Associate Professor in Operations Management at the Technical University of Denmark. He graduated from the University of Groningen in The Netherlands with a Ph.D. in Operations Management and an MSc in Econometrics and Operations Research. His (often interdisciplinary) research mostly deals with operations management in process industries, with topics ranging from planning and scheduling to supply chain design.

\section{ORCID}

Martin Grunow (1) http://orcid.org/0000-0002-5076-0169

Renzo Akkerman (1) http://orcid.org/0000-0001-8140-7643

\section{References}

Appelqvist, P., J.-M. Lehtonen, and J. Kokkonen. 2004. “Modelling in Product and Supply Chain Design: Literature Survey and Case Study." Journal of Manufacturing Technology Management 15 (7): 675-686. doi:10.1108/1741038041055 5916.

Baud-Lavigne, B., B. Agard, and B. Penz. 2012. "Mutual Impacts of Product Standardization and Supply Chain Design." International Journal of Production Economics 135: 50-60. doi:10.1016/j.ijpe.2010.09.024. 
Beamon, B. M. 1998. "Supply Chain Design and Analysis: Models and Methods." International Journal of Production Economics 55: 281-294. doi:10.1016/S0925-5273(98)00079-6.

Behncke, F. G. H., F. Walter, and U. Lindemann. 2014. "Procedure to Match the Supply Chain Network Design with a Product's Architecture." Procedia CIRP 17: 272-277. doi:10.1016/j.procir.2014.02.033.

Blackhurst, J., T. Wu, and P. O’Grady. 2005. “PCDM: A Decision Support Modeling Methodology for Supply Chain, Product and Process Design Decisions." Journal of Operations Management 23: 325-343. doi:10.1016/j.jom.2004.05.009.

Chiu, M. C., and G. Okudan. 2014. "An Investigation on the Impact of Product Modularity Level on Supply Chain Performance Metrics: An Industrial Case Study." Journal of Intelligent Manufacturing 25 (1): 129-145. doi:10.1007/s10845012-0680-3.

CNBC. 2018. https://www.cnbc.com/2018/04/18/tesla-bat tery-production-is-the-real-bottleneck-not-model-3-pro duction.html.

Colson, G., and C. de Bruyn. 1989. Models and Methods in Multiple Objective Decision Making, Models and Methods in Multiple Criteria Decision Making. Oxford, UK: Pergamon Press.

De Boer, L., E. Labro, and P. Morlacchi. 2001. "A Review of Methods Supporting Supplier Selection." European Journal of Purchasing \& Supply Management 7 (2): 75-89. doi:10.1016/S0969-7012(00)00028-9.

Dori, D. 2002. Object-Process Methodology: A Holistic Systems Paradigm. Berlin: Springer.

Duclos, L. K., R. J. Vokurka, and R. R. Lummus. 2003. "A Conceptual Model of Supply Chain Flexibility." Industrial Management and Data Systems 103 (6): 446-456. doi:10.1108/02635570310480015.

Ehrgott, M., J. R. Figuira, and S. Greco, eds. 2010. Trends of Multiple Criteria Decision Analysis. Berlin: Springer.

Eisenhardt, K. M., and M. E. Graebner. 2007. "Theory Building from Cases: Opportunities and Challenges." Academy of Management Journal 50 (1): 25-32. doi:10.2307/20159839.

Ellram, L. M., and L. L. Stanley. 2008. "Integrating Strategic Cost Management with a 3DCE Environment: Strategies, Practices, and Benefits." Journal of Purchasing and Supply Management 14: 180-191. doi:10.1016/j.pursup.2008.05. 003.

ElMaraghy, H. A., and N. Mahmoudi. 2009. "Concurrent Design of Product Module Structure and Global Supply Chain Configurations." International Journal of Computer Integrated Manufacturing 22 (6): 483-493. doi:10.1080/0951 1920802389553.

Feng, T., and F. Zhang. 2014. "The Impact of Modular Assembly on Supply Chain Efficiency." Production and Operations Management 23 (11): 1985-2001. doi:10.1111/poms.12182.

Fine, C. H. 1998. Clock Speed: Winning Industry Control in the Age of Temporary Advantage. Reading, MA: Perseus Books.

Fine, C. H., and D. E. Whitney. 1996. "Is the Make-Buy Decision Process a Core Competence?” Working Paper, MIT Center for Technology, Policy and Industrial Development, Cambridge, USA.

Fisher, M. 1997. "What is the Right Supply Chain for Your Product?" Harvard Business Review, March-April, 105-116.

Fixson, S. K. 2005. "Product Achitecture Assessment: A Tool to Link Product, Process, and Supply Chain Design
Decisions." Journal of Operations Management 23: 345-369. doi:10.1016/j.jom.2004.08.006.

Gan, T. S., and M. Grunow. 2016. "Concurrent Product and Supply Chain Design: A Literature Review, an Exploratory Framework and a Process for Modularity Design." International Journal of Computer Integrated Manufacturing 29 (12): 1255-1271. doi:10.1080/0951192X.2015.1067908.

Gershenson, J. K., G. J. Prasad, and Y. Zhang. 2004. "Product Modularity: Measures and Design Methods." Journal of Engineering Design 15: 33-51. doi:10.1080/09544820320001 01731.

Gokhan, N. M., K. L. Needy, and B. A. Norman. 2010. “Development of a Simultaneous Design for Supply Chain Process for the Optimization of the Product Design and Supply Chain Configuration Problem." Engineering Management Journal 22 (4): 20-30. doi:10.1080/10429247.2010.11431 876.

Gosling, J., L. Purvis, and M. M. Naim. 2010. "Supply Chain Flexibility as a Determinant of Supplier Selection." International Journal of Production Economics 128 (1): 11-21. doi:10.1016/j.ijpe.2009.08.029.

Guitouni, A., and J. M. Martel. 1998. "Tentative Guidelines to Help Choosing an Appropriate MCDA Method." European Journal of Operational Research 109: 501-521. doi:10.1016/S0377-2217(98)00073-3.

Hammer, M., G. Walder, L. Wech, U. Steininger, R. Eckl, M. Steffan, and M. Lienkamp. 2014. "Development of a Safe Energy Storage System for Small Electric Vehicles.” Paper presented at the Conference on Future Automotive Technology, Germany.

Helmer, R., A. Yassine, and C. Meier. 2010. "Systematic Module and Interface Definition Using Component Design Structure Matrix." Journal of Engineering Design 21 (6): 647-675. doi:10.1080/09544820802563226.

Henkel, J., and A. Hoffmann. 2018. "Value Capture in Hierarchically Organized Value Chains." Journal of Economics and Management Strategy doi:10.1111/jems.12278.

Ho, W., X. Xu, and P. K. Dey. 2010. "Multi-Criteria DecisionMaking Approaches for Supplier Evaluation and Selection: A Literature Review." European Journal of Operational Research 202 (1): 16-24. doi:10.1016/j.ejor.2009.05.009.

Hölttä, K., E. S. Suh, and O. de Weck. 2005. "Trade-Off between Modularity and Performance for Engineered Systems and Products." Proceeding of the ICED 05: 15th International Conference on Engineering Design: Engineering Design and the Global Economy, Engineers Australia, Melbourne.

Jayaram, J., and S. Vickery. 2018. "The Role of Modularity in the Supply Chain Context: Current Trends and Future Research Directions." International Journal of Production Research 56 (20): 6568-6574. doi:10.1080/00207543.2018.1484574.

Jung, S., and T. W. Simpson. 2017. "New Modularity Indices for Modularity Assessment and Clustering of Product Architecture." Journal of Engineering Design 28 (1): 1-22. doi:10.1080/09544828.2016.1252835.

Ketokivi, M., and T. Choi. 2014. "Renaissance of Case Research as a Scientific Method." Journal of Operations Management 32 (5): 232-240. doi:10.1016/j.jom.2014.03.004.

Khan, O., and A. Creazza. 2009. "Managing the Product Design-Supply Chain Interface: Towards a Roadmap to the 'Design Centric Business'." International Journal of Physical Distribution and Logistics Management 39 (4): 301-319. doi:10.1080/0020754031000120087. 
Kusiak, A., and W. S. Chow. 1987. "An Efficient Cluster Identification Algorithm." IEEE Transactions on Systems, Man, and Cybernetics 17 (4): 696-699. doi:10.1109/TSMC.1987.289 363.

Meixell, M. J., and V. B. Gargeya. 2005. "Global Supply Chain Design: A Literature Review and Critique.” Transportation Research Part E: Logistics and Transportation Review 41: 531-550. doi:10.1016/j.tre.2005.06.003.

Melo, M. T., S. Nickel, and F. Saldanha-da-Gama. 2009. "Facility Location and Supply Chain Management - a Review." European Journal of Operational Research 196: 401-412. doi:10.1016/j.ejor.2008.05.007.

Mikkola, J. H., and O. Gassmann. 2003. "Managing Modularity of Product Architectures: Toward an Integrated Theory." IEEE Transactions on Engineering Management 50 (2): 204-218. doi:10.1109/TEM.2003.810826.

Nepal, B., L. Monplasir, and O. Famuyiwa. 2012. "Matching Product Architecture with Supply Chain Design." European Journal of Operational Research 216: 312-325. doi:10.1016/j.ejor.2011.07.041.

Noori, H., and D. Georgescu. 2008. "Making Supply Chain Design the Rational Differentiating Characteristic of the OEMs." International Journal of Production Research 46 (10): 2765-2783. doi:10.1080/00207540601011527.

Novak, S., and S. D. Eppinger. 2001. "Sourcing by Design: Product Complexity and the Supply Chain." Management Science 47 (1): 189-204. doi:10.1287/mnsc.47.1.189.10662.

Pashaei, S., and J. Olhager. 2015. "Product Architecture and Supply Chain Design: A Systematic Review and Research Agenda." Supply Chain Management: An International Journal 20 (1): 98-112. doi:10.1108/SCM-12-2013-0487.

Pero, M., N. Abdelkafi, A. Sianesi, and T. Blecker. 2010. “A Framework for the Alignment of New Product Development and Supply Chains." Supply Chain Management: An International Journal 15 (2): 115-128. doi:10.1108/1359854101102 8723.

Ross, A. M., D. H. Rhodes, and D. E. Hastings. 2008. “Defining Changeability: Reconciling Flexibility, Adaptability, Scalability, Modifiability, and Robustness for Maintaining System Lifecycle Value." Systems Engineering 11 (3): 246-262. doi:10.1002/sys.20098.

Saleh, J. H., G. Mark, and N. C. Jordan. 2009. "Flexibility: A Multi-Disciplinary Literature Review and a
Research Agenda for Designing Flexible Engineering Systems." Journal of Engineering Design 20 (3): 307-323. doi:10.1080/09544820701870813.

Simchi-Levi, D. 2013. Operations Rules. Cambridge, MA: MIT Press.

Suh, N. P. 1998. "Axiomatic Design Theory for Systems." Research in Engineering Design 10: 189-209. doi:10.1007/s00 1639870001.

Tang, C., and B. Tomlin. 2008. "The Power of Flexibility for Mitigating Supply Chain Risks." International Journal of Production Economics 116 (1): 12-27. doi:10.1016/j.ijpe.2008.07. 008.

Tilstra, A. H., C. C. Seepersad, and K. L. Wood. 2012. "A High-Definition Design Structure Matrix (HDDSM) for the Quantitative Assessment of Product Architecture." Journal of Engineering Design 23 (10-11): 767-789. doi:10.1080/09544828.2012.706748.

Uiterwijk, D., J. Soeters, and P. van Fenema. 2013. "Aligning National 'logics' in a European Military Helicopter Program." Defense \& Security Analysis 29 (1): 54-67. doi:10.1080/14751798.2013.760248.

Ulrich, K. T. 1995. "The Role of Product Architecture in the Manufacturing Firm." Research Policy 24 (3): 419-440. doi:10.1016/0048-7333(94)00775-3.

Voss, C., N. Tsikriktsis, and M. Frohlich. 2002. "Case Research in Operations Management." International Journal of Operations \& Production Management 22 (2): 195-219. Doi:10.1108 /01443570210414329.

Walden, D. D., G. J. Roedler, K. J. Forsberg, R. D. Hamelin, and T. M. Shortell, eds. 2015. INCOSE Systems Engineering Handbook: A Guide for System Life Cycle Processes and Activities, 4th Edition. Hoboken, New Jersey: John Wiley \& Sons.

Yao, X., and R. Askin. 2019. "Review of Supply Chain Configuration and Design Decision-Making for new Product." International Journal of Production Research 57 (7): 2226-2246. Doi: 10.1080/00207543.2019.1567954.

Zhang, X., G. Q. Huang, and M. J. Rungtusanatham. 2008. "Simultaneous Configuration of Platform Products and Manufacturing Supply Chains." International Journal of Production Research 46 (21): 6137-6162. doi:10.1080/00207540 701324150 . 